\title{
Impairing Proliferation of Glioblastoma Multiforme with CD44+ Selective Conjugated Polymer Nanoparticles
}

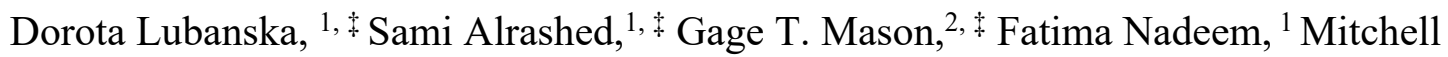
DiPasquale, ${ }^{2}$ Angela Awada, ${ }^{2}$ Aleena Malik, ${ }^{2}$ Mohamed A.R. Soliman, ${ }^{3,}{ }^{4}$ Ana C. deCarvalho, ${ }^{5}$ Abdalla Shamisa, ${ }^{4}$ Swati Kulkarni, ${ }^{4}$ Drew Marquardt, ${ }^{2,6}$ Lisa A. Porter ${ }^{1, *}$ and Simon RondeauGagné ${ }^{2, *}$

${ }^{1}$ Department of Biomedical Sciences, University of Windsor, 401 Sunset Ave. Windsor, Ontario, Canada N9B 3P4 ${ }^{1}$

${ }^{2}$ Department of Chemistry and Biochemistry, Advanced Materials Centre of Research (AMCORe), University of Windsor, 401 Sunset Ave. Windsor, Ontario, Canada N9B 3P4

${ }^{3}$ Department of Neurosurgery Faculty of Medicine Cairo University Cairo, Egypt

${ }^{4}$ Schulich School of Medicine, Western University, London, ON, Canada

${ }^{5}$ Department of Neurosurgery, Henry Ford Hospital, Detroit, MI 48202, USA

${ }^{6}$ Department of Physics, University of Windsor, 401 Sunset Ave. Windsor, Ontario, Canada N9B 3P4

$\$$ These authors have equally contributed to this work

* Co-corresponding authors

Glioblastoma is one of the most aggressive types of cancer with median survival of only 15 months. Successful therapy is hampered by the existence of treatment resistant populations of stem-like tumour initiating cells (TICs) and poor blood-brain barrier drug penetration. Therapies capable of effectively targeting the TIC population are in high demand. Here, we synthesize spherical diketopyrrolopyrrole (DPP)-based conjugated polymer nanoparticles (CPNs) with an average diameter of $109 \mathrm{~nm}$. The CPN were designed to include fluoresceinconjugated hyaluronic acid (HA), a ligand for the CD44 receptor present on one population of TICs. We demonstrate blood-brain barrier permeability of this system and concentration and cell cycle phase-dependent selective uptake of HA-CPNs in CD44 positive GBM-patient derived cultures. Interestingly, we found that uptake alone decreases stemness, invasive properties and proliferation of the CD44-TIC population in zebrafish PDX models in vivo. 


\section{This study is the first to show surface moiety-driven selectivity of conjugated polymer nanoparticles in targeting TIC populations in brain cancer.}

\section{Introduction}

Glioblastoma, frequently abbreviated as GBM, is the most aggressive type of brain tumour with survival of less than 15 months after diagnosis. ${ }^{1,2}$ The aggressive and therapy resistant nature of GBM is attributed in part to the characteristic intra- and inter- tumoural heterogeneity of the disease. ${ }^{3,4}$ A plethora of genetic aberrations contribute to the unique evolution of the tumour mass in individual patients and is a consequence of the uncontrolled activation of diverse molecular pathways driving GBM invasion, progression, and therapy resistance. ${ }^{5}$ At the cellular level, heterogeneity of GBM tumours can be described by a hierarchical model, with populations of immature tumour initiating cells (TICs) feeding the GBM growth, treatment resistance, and recurrence. ${ }^{6}$ TICs have been extensively characterized in literature by the expression of several cell surface markers, including a trans-membrane glycoprotein receptor, $\mathrm{CD} 44 .{ }^{7} \mathrm{CD} 44$ is activated by binding to its primary ligand hyaluronic acid (HA) to trigger downstream signaling via several pathways including the activation of Akt and mitogen activated protein kinase (MAPK) cascades. ${ }^{8}$ CD44 activation results in enhanced cell proliferation and migration, ${ }^{9,10}$ and expression levels correlate negatively with survival times in GBM patient populations, confirmed recently in a large scale meta-analysis study. ${ }^{11,12}$ While CD44 is not the only marker of the TIC population, it represents an attractive target to begin the design of novel targeted therapies against this aggressive initiating cell population within GBM. ${ }^{6}$

Developing novel therapies capable of passing the blood-brain barrier (BBB) is an additional challenge required to effectively target the TIC population. ${ }^{13,14} \mathrm{~A}$ variety of mechanisms 
of transport across the $\mathrm{BBB}$ have been described in literature, ${ }^{15,16}$ pointing at the limited permeability potential and call for therapeutic solutions that could overcome this limitation. The use of nanoparticles (NP) may serve as an effective therapeutic modality for GBM; however, various obstacles need to be taken into consideration, such as nanoparticle size and potential chemistry-mediated toxicity. Conjugated polymer nanoparticles (CPNs) are an intriguing class of nanomaterials prepared from rigid, semi-crystalline semiconducting polymers. ${ }^{17-19}$ Due to their remarkable optical and electronic properties, CPNs have generated a great amount of interest in recent years for the design and preparation of new theranostic agents. ${ }^{20}$ Furthermore, previous studies have shown that CPNs have a high photothermal conversion efficiency and can be used for photoacoustic imaging. ${ }^{21,22}$ Given the characteristics of their potential benefit to anti-cancer strategies and consideration as an effective anti-glioma therapy, it is desired that CPNs exhibit a targeting specificity through design-incorporated recognition units for specific molecules on the surface of TIC populations.

Several studies provided evidence, on a wide range of nanomaterials, that the presence of recognition units yielded effective targeting activity and increased their anti-glioma properties; ${ }^{23-}$ 25 they often presented highly complexed designs and synthesis, thereby introducing potential obstacles in bioavailability or large-scale production which has impeded translational application. HA offers promise as a low complexity recognition unit when incorporated into inorganic and organic nanomaterials, offering active targeting and stimuli-responsive degradation in diverse diseases. ${ }^{26,27}$ The role of HA in targeting specific populations of TICs in glioblastoma using conjugated polymer- based nanoparticles has not been explored to date.

Herein, we report the design, preparation, and structural characterization of novel conjugated polymer nanoparticles generated through nanoprecipitation of a diketopyrrolopyrrole 
(DPP)-based conjugated polymer with a fluorescein-tagged HA co-polymer (HA-CPNs). Careful and detailed evaluation using a plethora of in vitro and PDX in vivo assays demonstrates bloodbrain penetration of this nano-system in zebrafish, and CD44-selective and anti-proliferative effects on human glioma. The obtained results confirm that these new CPNs are a strong candidate for further validation to be used in novel targeting therapies against GBM and could provide the foundation for a platform of conjugated polymer targeted therapeutics for stem cell driven cancers like GBM.

\section{Results}

Structural characterization of HA-CPNs. In this study, a DPP-based conjugated polymer, $\mathrm{P}(\mathrm{DPP}-\mathrm{T})$, was prepared according to previously reported literature (Figure S1, S2 and S3). ${ }^{28}$ Following its synthesis, P(DPP-T) was used with commercially available fluorescein-labelled hyaluronic acid to prepare the HA-CPNs via nanoprecipitation (Fig.1a). To confirm the size and to further unveil the morphological characteristics of HA-CPNs, various characterization techniques were utilized. First, the CPNs were evaluated by small angle neutron scattering (SANS). This technique, commonly used for the characterization of vesicles and lipid selfassembly, is a particularly valuable technique to probe for the shape of nanoparticles as measurements can be carried out directly in a free-floating suspension or solution without further manipulations. Scattering form factors were fit using a generalized Guinier-Porod model for an unbiased assessment of particle structure following established methodology (Figs.1b-d). ${ }^{29,30}$ This analysis gives insight to the shape and size of particles based on the Porod exponent $(d)$, dimensionality $(s)$, and radius of gyration $\left(R_{g}\right)$. Fits to HA-CPNs yielded $d=4.2 \pm 0.02$ and $\mathrm{s}=$ $0.02 \pm 0.06$, which strongly correlates to a hard sphere with a smooth surface $(d=4$ and $s=0) .{ }^{29}$ 
The extracted $R_{g}=32.6 \pm 0.8 \mathrm{~nm}$ was extended to determine the real-space cross-sectional radius $\left(R=R_{g}(5 / 3)^{1 / 2}\right)$ of HA-CPNs to be $42.1 \pm 1.0 \mathrm{~nm}$. To confirm the results obtained by SANS, dynamic light scattering (DLS) was used, with the results depicted in Fig.1e. A polydispersity index of 0.432 was determined, which confirms a relatively uniform distribution of sizes with an average hydrodynamic radius $\left(R_{h}\right)$ of $54.5 \mathrm{~nm}$. To gain further insight on the geometry of the nanoparticles, the $R_{g} / R_{h}$ value can also be evaluated. Based on the literature, a $R_{g} / R_{h}$ ratio $<0.775$ can be associated to hard spheres. Therefore, this result confirms the formation of spherical nanoparticles, as previously demonstrated for similar systems. The structure, average diameters and spherical shape of the new CPNs were again corroborated by transmission electron microscopy (TEM) as shown in Fig.1f. 

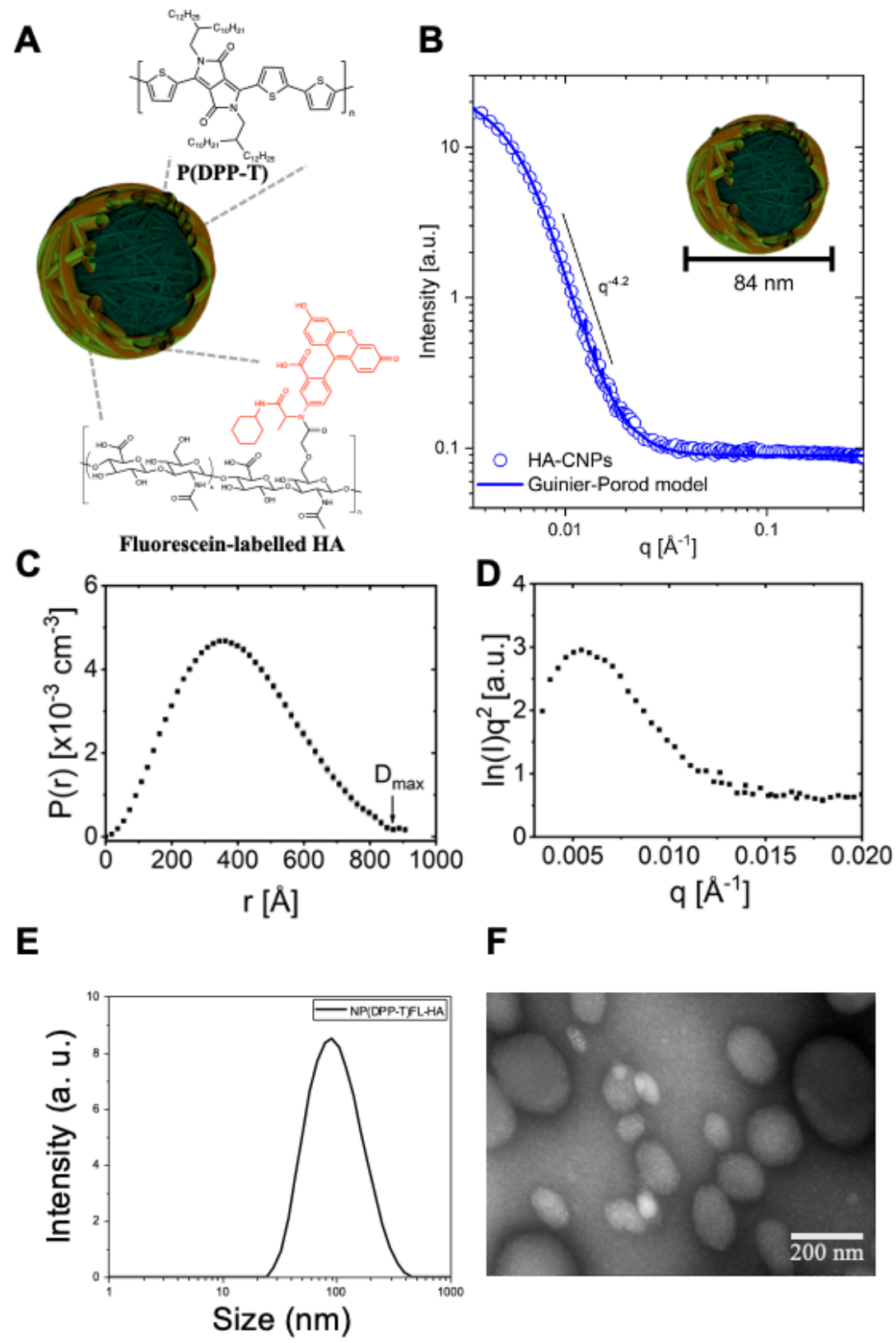

F

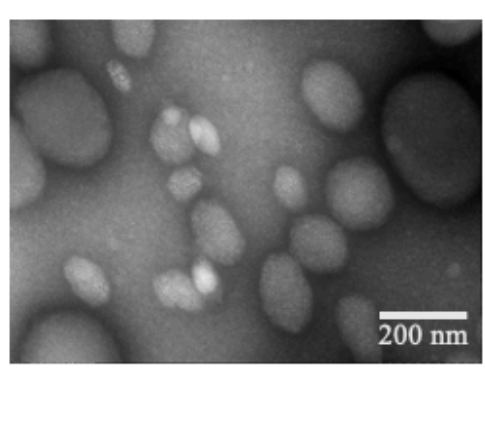

Fig. 1 Design and Structural Characterization of the HA-CPNs. a Schematic structure of HACPNs; b SANS form factor for a dilute dispersion of HA-CNPs in $\mathrm{D}_{2} \mathrm{O}$ fit with a solid line using an empirical Guinier-Porod model. The slope in the Porod region of the plot shows a q-dependency of $\mathrm{q}^{-4.2}$, suggesting a spherical particle shape; $\mathbf{c}$ Real-space distance distribution function from an inversion approach demonstrating uniformity consistent with spherical particles of maximum dimension $\mathrm{D}_{\max } ; \mathbf{d}$ Kratky plot demonstrates a peak and plateau characteristic of compact globular 
structures. e Dynamic light scattering (DLS) of HA-CPNs. Average diameter of $109 \mathrm{~nm}$; f Transmission electron microscopy (TEM) image of HA-CPNs. Scale bar of $500 \mathrm{~nm}$.

Optimization and effects of HA-CPN uptake by glioma cells. To study the effects of HA-CPNs on glioma cell biology it is essential to determine the optimal concentration of the particles and treatment timing allowing for the HA-CPN uptake in vitro. The U-251 MG glioma cells were treated with HA-CPNs at the concentration of 12, 24 and 36 CPNs per cell over 48 hours and the number of CPN-positive cells were measured at the indicated time points using flowcytometry (Fig.S4a). We found the HA-CPN uptake to be time and concentration dependent with significantly upregulated number of HA-CPN positive cells at 48 and 24 hours of treatment using both 24 and/or 36 particles per cell in comparison to the 12 particles/cell incubation. Subcellular localization analysis via microscopy at 3, 24 and 48 hours revealed that HA-CPNs localize primarily at the cell membrane and cytoplasm (Fig.S4b). Treatment of U-251 MG cells with further increased HA-CPN concentrations over 96-hour time period showed $95 \%$ saturation of cell population with the nanoparticles when cultures were treated with 96 particles/ cell (Fig.S4c).

Furthermore, we found that when the same number of U-251 MG cells were treated with $5,10,24,48,60 \mathrm{HA}-\mathrm{CPNs} / \mathrm{cell}$ over a time course of 6 days, the concentrations tested, up to $\sim 24$ HA-CPNs/cell, did not affect cell proliferation (Fig.S5a). Surprisingly, however, a statistically significant decrease of the cell number was found at the concentrations of 48 and 60 HACPNs/cell, when compared to the vehicle control (Fig.S5a). To assess whether the exposure of glioma cells to HA-CPNs influences GBM metabolic activity in vitro, we subjected U-251 MG cell line to MTT assay in the presence of the same particles/cell concentration range as for the proliferation assay. A significant decrease in metabolic activity relative to the vehicle control at 
72 hours, was found for cultures treated with 24, 48, and 60 HA-CPNs/cell (Fig.S5b). The results demonstrated that HA-CPN uptake by glioma cells is time and concentration dependent and the exposure to HA-CPNs at higher concentration results in cytostatic effects.

\section{HA-CPN uptake is cell cycle phase- dependent.}

It has been demonstrated previously that the efficiency of the nanoparticle uptake can be dependent on the phase that cells are in within the cell cycle. ${ }^{31}$ To determine the impact of cell cycle phase on the uptake of tested nanoparticles, U-251 MG cells were synchronized in select cell cycle phases. HA-CPNs at 24 particles per cell, were introduced for 3 hours into the synchronous cultures and the uptake was analyzed using flowcytometry. Enrichment in G0/G1 was accomplished using double thymidine block. Starvation ( $0 \%$ FBS, $24 \mathrm{~h})$ resulted in approximately $70 \%$ of cells to reside in $\mathrm{G} 1 / \mathrm{G} 0$ and over $11 \%$ and $16 \%$ in S and $\mathrm{G} 2 / \mathrm{M}$ phases, respectively. The enrichment in $\mathrm{G} 2 / \mathrm{M}$ phase was accomplished upon the treatment with nocodazole $(2 \mathrm{pg} / \mathrm{mL})$ (Fig.S6a). We found that mitotic populations of cells had the highest number of cells positive for HA-CPNs, when compared to starvation and thymidine treated cultures, accounting for $70 \%$ of the population tested (Fig.S6b).

\section{HA-CPNs demonstrate selective uptake and anti-proliferative properties in vitro. To} determine whether the HA molecules, present on CPNs, mediate uptake of the nanoparticles in glioma cells in vitro, U-251 MG cells were incubated with HA-containing conjugated polymer nanoparticles (HA-CPNs) and conjugated polymer nanoparticles prepared from polysorbate that do not contain HA (Nc-CPNs) as control (see Supplementary Information for details). CPNs

possess weak innate fluorescence $(524 \mathrm{~nm})$ which allowed for signal quantification and 
comparative analysis in this experiment using same excitation/emission spectrum for both treatments. The cells were treated with 24 CPNs per cell for 1 hour, washed, and the innate fluorescent signal was quantified at the indicated time points using a plate reader. We found an increase in fluorescence at 0.5 -hour and 1-hour timepoint when cells were treated with HA-CPNs in comparison to Nc-CPN treatment at the same timepoints (Fig.2a). To address the potential selectivity of HA-CPNs towards glioma populations characterized by CD44 expression, U-251 MG cells were incubated with increasing concentrations of HA-CPNs for 48 hours followed by staining with a fluorescent anti-CD44 antibody. The HA-CPN uptake was monitored using microscopy (Fig.2b). We found the HA-CPNs to be present at the cell membrane and it the cytoplasm (Fig.2b). The number of HA-CPN positive cells in relation to CD44 expression was assessed using flowcytometry (Fig.2c). At 24, 48, and 96 HA-CPNs/cell concentration tested, there was significantly higher upregulation $(\sim 8, \sim 16$ and $\sim 13$-fold, respectively) of the HA-CPN uptake by populations staining positively for CD44 in comparison to CD44 negative cells with (Fig.2c). Consequently, 24- hour HA-CPN treatment of FACS- derived CD44+ and CD44- U-251 MG cells (Fig.2d, left) revealed that CD44+ cells demonstrated significantly increased enrichment for the nanoparticles in comparison to CD44- cells at 24 and 36 HA-CPNs/cell (Fig.2d, right). To determine whether the selective uptake of HA-CPNs by CD44+ glioma populations can impact their proliferative potential, CD44-, CD44+ and heterogeneous populations of U251 cells were treated with HA-CPNs for 7days and sorted based on the HA-CPN content. CD44-, CD44+ and heterogeneous - CPN positive and CPN negative cultures were subjected to the proliferation assay. HA-CPN uptake was selectively downregulating proliferation of CD44+ cells with CD44+ cells enriched for HA-CPNs demonstrating the most significant decrease of proliferation (Fig.2e). 
Highly propagated and commercially available cell lines fail to mimic the actual state of the disease and may produce false results in functional assays and therapy response screens, in vitro and in vivo. To further examine the selectivity of the nanomaterials system, using liferelevant model, we employed cell cultures derived from tumours obtained at the time of surgery, from individual GBM patients. Five GBM patient- derived cultures (\#1-\#5) were treated with HACPNs for 7 days, stained with CD44 antibody and the uptake was analyzed via flowcytometry. Four out of five cultures tested showed significant upregulation of the HA-CPN uptake in CD44+ cells (Fig. 2f) further validating the use of this type of nanoparticles in potential, novel TICtargeting therapies against glioma. 

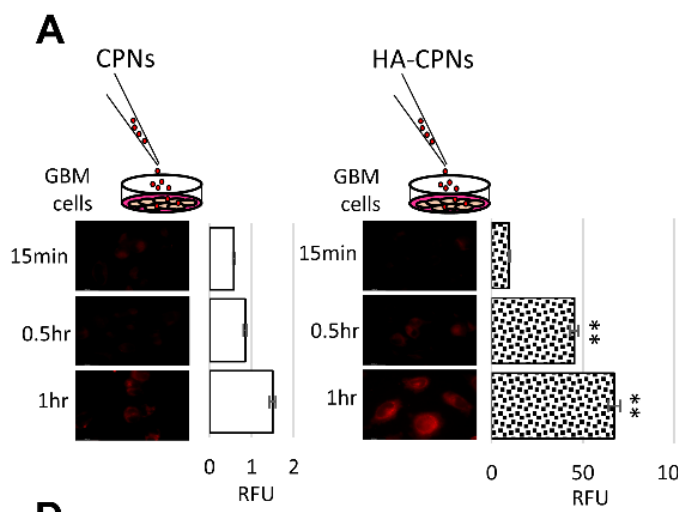

B

C
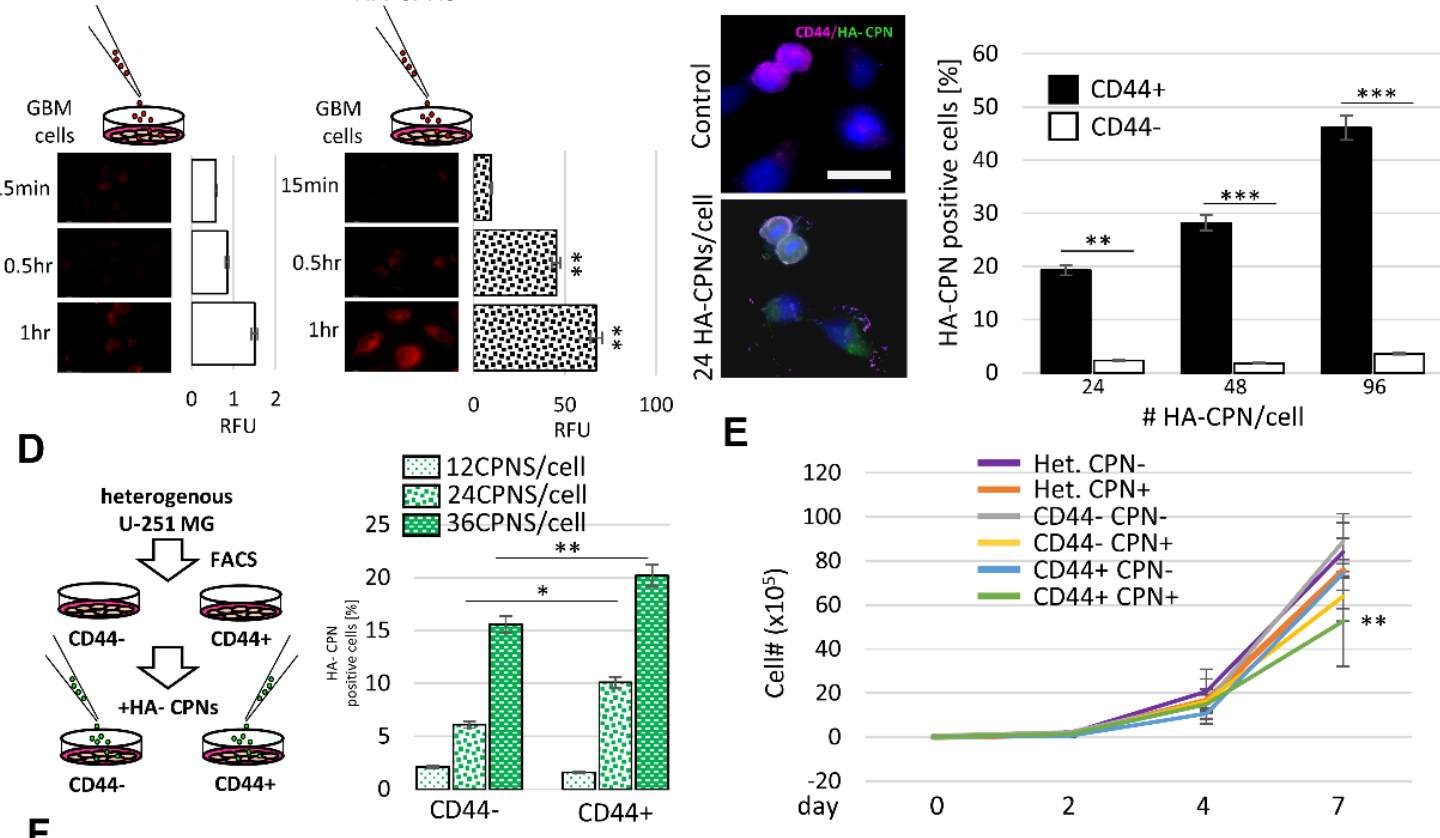

$\mathbf{F}$

E
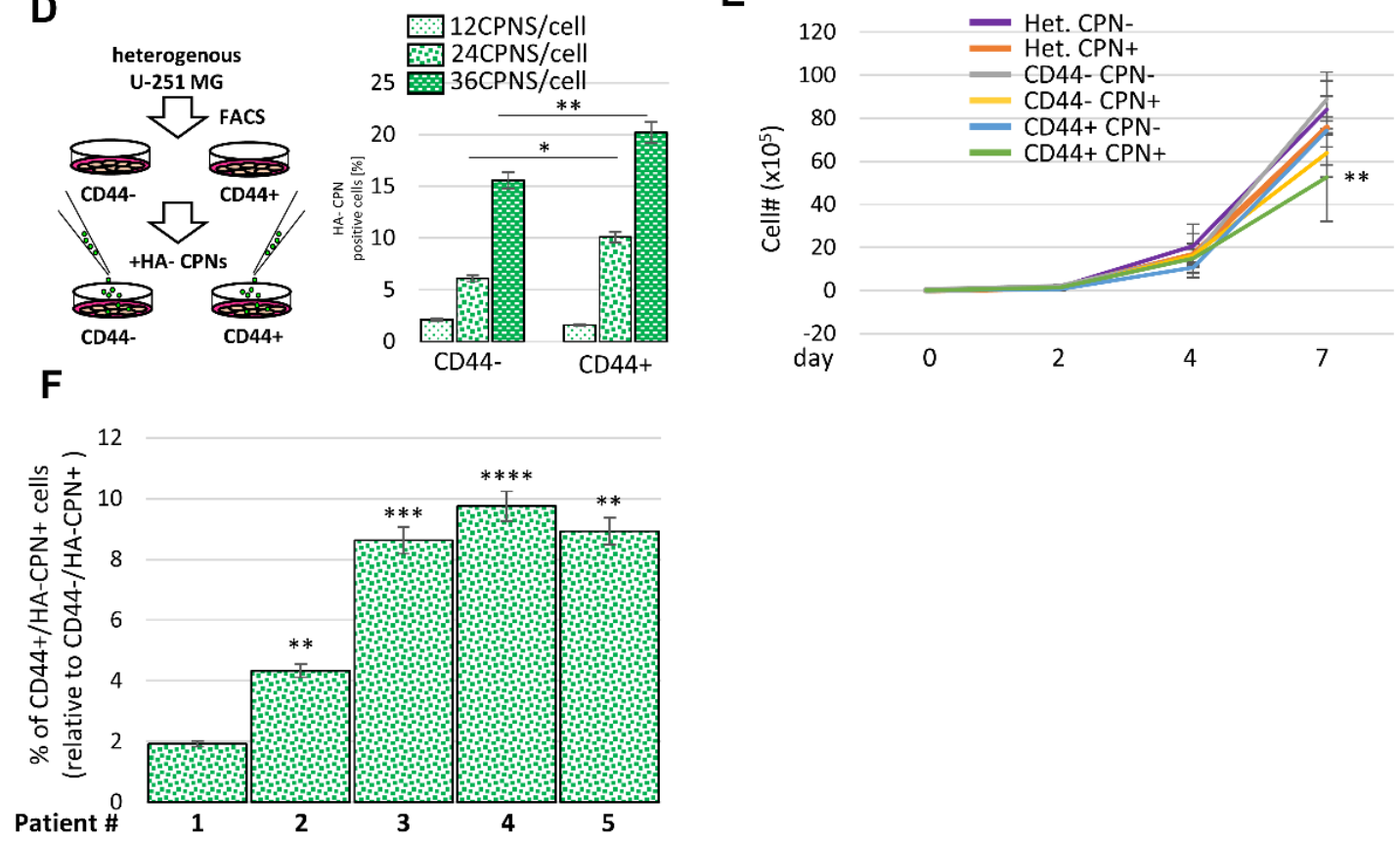

Fig. 2 HA-CPNs demonstrate selective uptake by CD44 enriched U-251 MG cells as well as GBM patient- derived cells. a Quantified fluorescence (RFU) in U251 MG cells treated with HACPNs and CPNs without conjugated HA, at the indicated time points using a plate reader. b Representative images of cells stained with anti-CD44 antibody and treated with $24 \mathrm{HA}-\mathrm{CPNs} / \mathrm{cell}$ or vehicle control (Control); scale bar: $25 \mu \mathrm{m}$. c flowcytometry analysis of U251 MG cells stained with anti-CD44 antibody and treated with 24, 48, 96 HA-CPNs/cell or vehicle control (Control). Cells positive for HA-CPNs graphed as \% of cells positive (CD44+) and/or negative (CD44-) for the antibody stain. d Flowcytometry analysis of HA- CPN positive U251 MG cells in FACS- 
derived CD44+ and CD44- populations treated with 12, 24, 36 HA-CPNs/cell graphed as \% of the population tested. e Proliferation of heterogeneous (Het.), CD44+ and CD44- populations of U$251 \mathrm{MG}$ cells, FACS- enriched for HA-CPNs (HA-CPN+) compared to HA-CPN- cells, assessed using trypan blue exclusion assay. f GBM patient- derived cultures (\#1-\#5) tested for CD44selective HA-CPN uptake and quantified as $\%$ of CD44+ cells relative to the $\%$ uptake in CD44cells. Data shown as mean $\pm \mathrm{s} . \mathrm{d}, \mathrm{n}=3,{ }^{*} \mathrm{p}<0.05,{ }^{* *} \mathrm{p}<0.01,{ }^{* * *} \mathrm{p}<0.001$; Student's $t$-test.

\section{Proliferation and stemness of primary GBM patient derived cells are decreased by HA-CPN}

treatment. To test the impact of HA-CPNs on glioma proliferative potential in cells derived from GBM tumours, primary cultures (HF3035, HF2927, HF2303) were treated with HA-CPNs along with the vehicle control. Trypan blue exclusion assay demonstrated a significant decrease of proliferation in two, out of three patient- derived cultures treated with $24 \mathrm{HA}-\mathrm{CPNs} / \mathrm{cell}$ and/or vehicle control. At 12 hour- timepoint, the proliferation of HF3035 and HF2927 was inhibited by $19.3 \%$ and $31.5 \%$, respectively, when incubated with HA-CPNs as compared to the vehicle control (Fig.3a) which could be attributed to the observed average decrease of viability in those lines (Fig.3b). Both lines demonstrated an average of $22 \%$ decrease of proliferation at 6 hours which was consistent with observed decreased proliferation in U251 MG cells treated with 24 HACPNs/cell for the same period of time (Fig. S5A). Given that HA-CPNs selectively target CD44+ TICs, neurosphere formation assay was performed to determine if the nanoparticle treatment can regulate stemness in GBM patient derived cell lines. We showed that primary GBM cells treated with HA-CPNs produced significantly lower number of spheres in two consecutive generations of spheres (primary and secondary) (Fig.3c) and the generated spheres were of smaller diameter (Fig.3d). Consequently, qRT-PCR analysis of well- established stemness markers showed 
significantly downregulated mRNA expression levels of PCNA, NANOG and upregulated levels of $M A P 2$, differentiation marker, in patient derived cultures, treated with HA-CPNs in comparison to the vehicle control (Fig.3e). We also observed significant decrease of BCL2A1, an antiapoptotic marker. The mRNA levels of MDR1, a crucial TIC- related drug resistance marker, remained unaffected by the HA-CPN treatment (Fig.3e). The obtained data show that HA-CPNs not only negatively regulate proliferation of glioma in primary patient cultures but also decrease stemness of GBM cells, suggesting that treatment with HA-CPNs can better the treatment, not only as a potential therapy "carrier" via selective binding of TICs but also through direct regulation of TIC aggressiveness. 


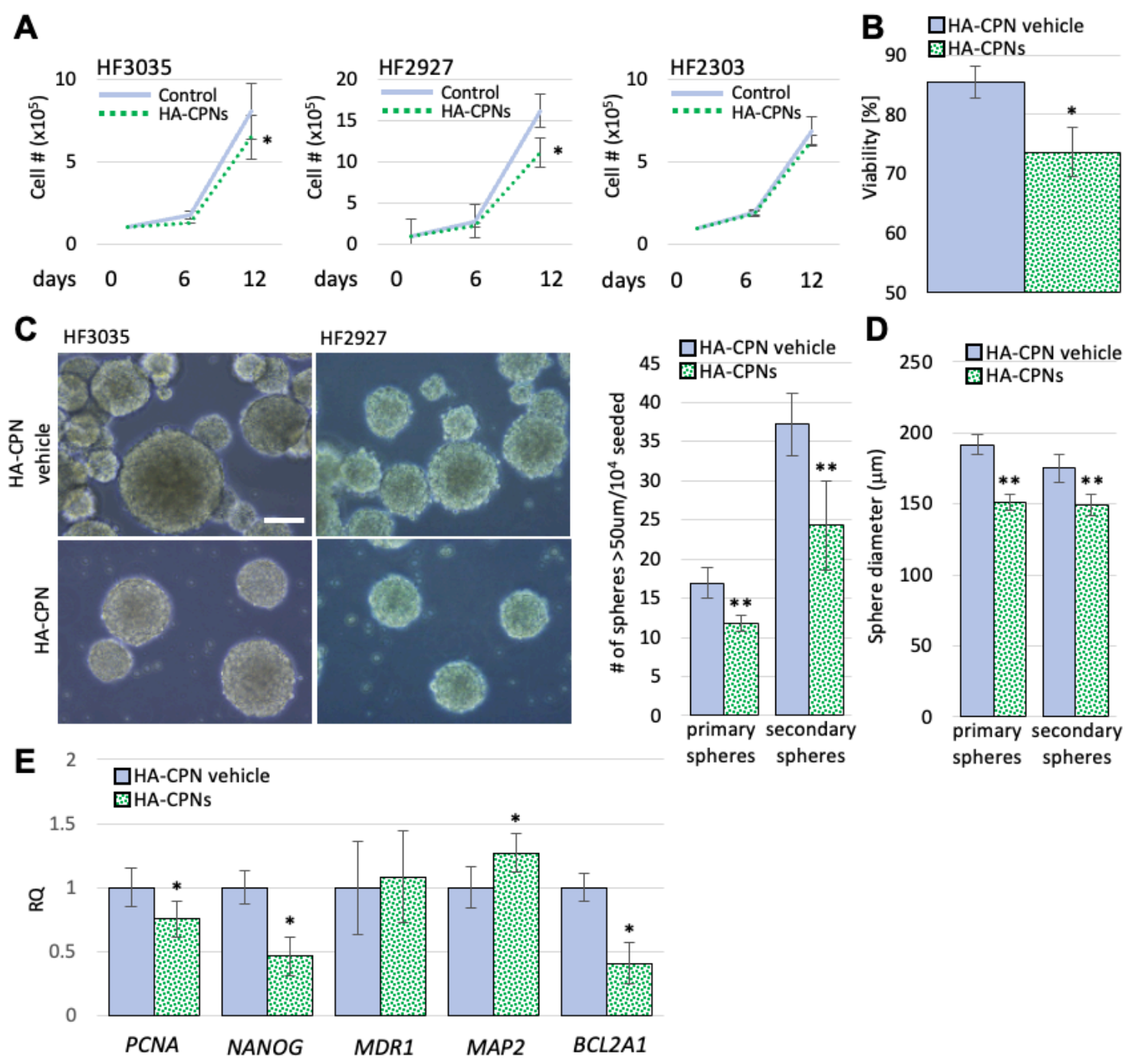

Fig.3. Effects of HA-CPNs on proliferation and stemness of primary GBM patient- derived cell lines. HF3035, HF2927 and HF2303 were treated with 24 HA-CPNs/ cell and/or HA-CPN vehicle control and subjected to the following assays. a Proliferation over the time course of 12 days. Cell number assessed via trypan blue exclusion assay at the indicated time points. b Average number of viable cells, over three cell lines tested, and assessed as \% of total population tested via trypan blue exclusion assay. c Neurosphere formation assay, representative images of HF3035 and HF2927 (left); scale bar $=100 \mu \mathrm{m}$. Number of spheres formed is quantified per every $10^{4}$ cells seeded, three lines averaged (right). d Average diameter of spheres formed; three lines pooled for 
analysis. e qRT-PCR analysis of mRNA levels of the indicated markers of stemness/differentiation. Data shown as mean $\pm \mathrm{s.d}, \mathrm{n}=3,{ }^{*} \mathrm{p}<0.05,{ }^{*} \mathrm{p}<0.01$; Student's $t$-test.

\section{Migration and invasion of GBM is negatively regulated in HA-CPN treated cultures.}

To further investigate the influence of the HA-CPNs on glioma aggressiveness, we analyzed the migratory and invasive properties of patient derived GBM cells treated with the nano-system. CD44 positive cells enriched for HA-CPNs via FACS (HA-CPN+) were used in parallel to FACSderived CD44+ cells negative for HA-CPN uptake (HA-CPN-). Boyden chamber assay showed that HA-CPN+ cells migrated significantly less in comparison to HA-CPN- cells (Fig.4a). Given the observed antiproliferative effect of HA-CPNs on glioma and to validate the migration assay results, we set up an invasion time course using single spheres generated from primary patient cells embedded in Matrigel, supplemented with either vehicle control or 24 HA-CPNs/cell (Fig.4b). We demonstrated that presence of HA-CPNs in the Matrigel significantly decreased the distance of the collective invasion in primary patient cells as compared to the vehicle control, over a time course of 6 days (Fig.4b). The analysis of invading single cells revealed that significantly more cells detached from the spheres were found when Matrigel ${ }^{\mathrm{TM}}$ supplemented with the vehicle control was used as compared to HA-CPN- supplemented Matrigel ${ }^{\mathrm{TM}}$ (Fig.4b, images\&Fig.4c). In comparison to the vehicle control presence, the single cells showed significantly shorter distance of invasion in the presence of HA-CPNs (Fig.4d). The demonstrated data confirms that HA-CPNs play a role in suppressing major hallmarks of GBM aggressiveness. 

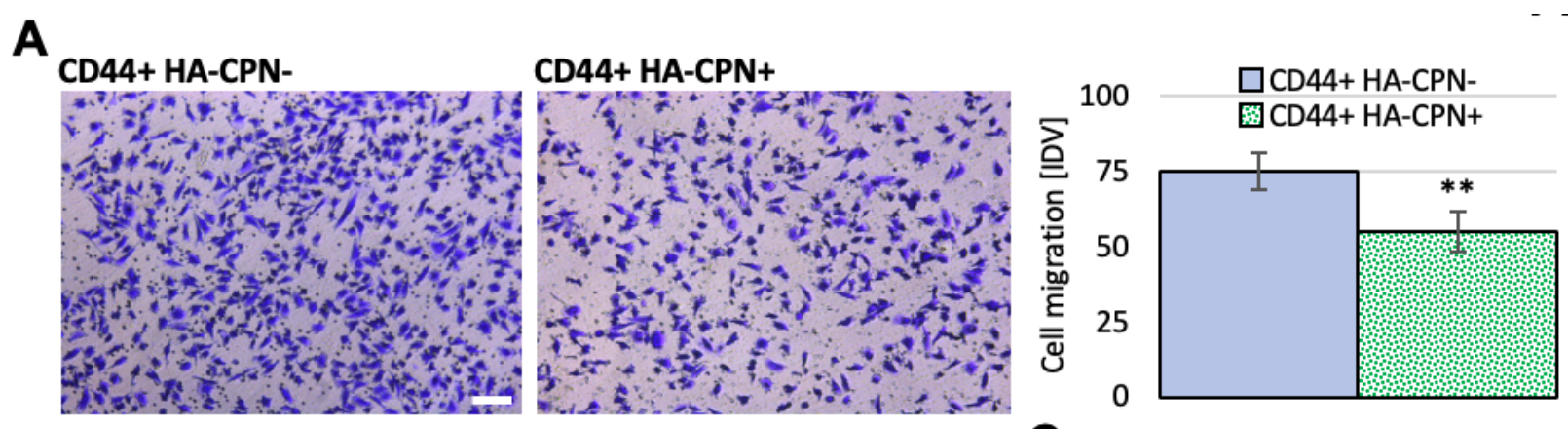

B
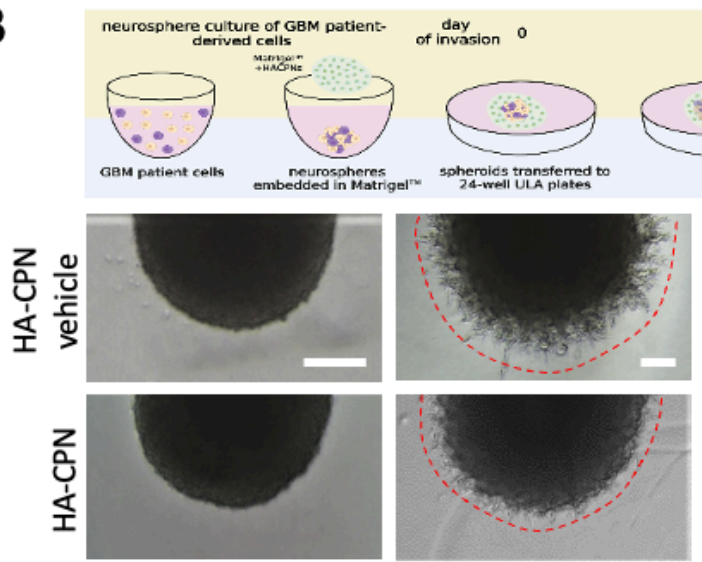

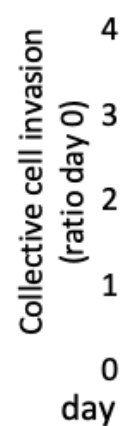

$\square$ HA-CPN vehicle 圈HA-CPNs

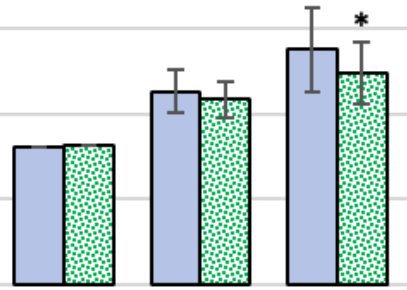

1

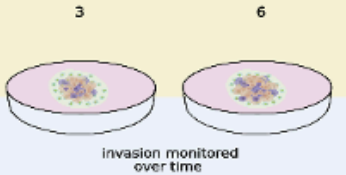

invasion monitored
overt time
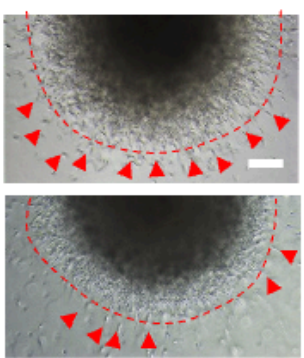

D

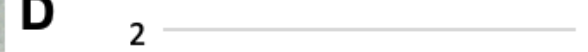

Fig. 4 Treatment with HA-CPNs regulates migration and invasion in glioma. a Boyden chamber assay using CD44+ U-251 MG cells treated and FACS- enriched for HA-CPNs (CD44+ HA-CPN+) compared to HA-CPN- cells (CD44+ HA-CPN-). Representative images (left). Scale bar $=100 \mu \mathrm{m}$. Migrated cells scored in five fields of view per replicate over three replicates, using ImageJ as Integrated Density Value (IDV) (right). b\&c Matrigel ${ }^{\mathrm{TM}}$ invasion assay using GBM patient derived spheres treated with HA-CPNs and/or HA-CPN vehicle control over a time course of six days. Schema of the protocol (top), representative images (mid panel). Scale bars $=100 \mu \mathrm{m}$. b Collective invasion measured using ImageJ as change in sphere radius over time. Quantified 
values averaged over 6 spheres per treatment, per time point, over 3 patient cell lines (bottom). Leading edge assessed optically, marked by dashed line. c Average number of single cells migrating (red arrowheads in b) scored using ImageJ per each Matrige ${ }^{\mathrm{TM}}$ - embedded sphere at the indicated timepoints. $\mathbf{d}$ Average distance of the single cell migration at the indicated timepoints. Data shown as mean $\pm \mathrm{s} . \mathrm{d}, \mathrm{n}=3,{ }^{*} \mathrm{p}<0.05,{ }^{* *} \mathrm{p}<0.01,{ }^{* *} \mathrm{p}<0.001$; Student's $t$-test.

\section{HA-CPNs penetrate blood-brain barrier in zebrafish and demonstrate selectivity for CD44+}

glioma cells in vivo. The inability to penetrate the blood- brain barrier by therapeutic agents is one of the biggest obstacles in an effective and lasting treatment of GBM. Hence, an effective therapy of GBM via systemic administration of HA-CPNs is dependent their ability to efficiently cross the blood- brain barrier. To assess whether the BBB is permeable to HA-CPNs, we injected the nanoparticles into the yolk sac of zebrafish embryos at 48 hours post fertilization (hpf) and analyzed their biodistribution over a period of two weeks. We found that at 5 hours post injection (hpi) HA-CPNs entered the duct of Cuvier inside the yolk sac and became clearly detectable with increasing levels in the head/brain area at 8hpi and 24hpi (Fig.5a). HA-CPNs were still present at quantifiable levels at 24hpi and 14 days post injection (dpi) (Fig.5a).

To investigate the effects and selectivity of HA-CPNs in glioma proliferation in vivo, zebrafish embryos at 48hpf were injected with FACS- derived CD44+ and/or CD44- U-251 MG populations in the presence of HA-CPNs and/or vehicle control (Fig.5b). At least fifty embryos per treatment were imaged at 5dpi (Fig.5c) and subjected to fluorescent signal quantification (Fig.5c, right). Comparative analysis revealed a significant decrease of the CD44+ but not CD44foci burden at day 5 of treatment when compared to U-251 MG cell burden at injection (0dpi) (Fig.5c, right). To investigate the tumour burden kinetics in the presence of HA-CPNs or vehicle 
control, 2dpf zebrafish embryos were injected with the co- suspension of CD44+ and CD44- U$251 \mathrm{MG}$ cells labeled with different fluorescent cell tracers in the presence of the tested nanoparticles and/or vehicle control. Foci formation was monitored over time and images were taken at the indicated time points (Fig.5d, left). We found that the extent of tumour burden from co-injected populations in the presence of HA-CPNs was significantly decreased for CD44+ derived foci but not for CD44- ones at 24hpi and 4dpi of the time course (Fig.5d, right). The obtained data demonstrated that HA-CPNs are able to pass through the blood-brain barrier in zebrafish embryos and decrease tumour burden through selective targeting of CD44+ TICs. 
A
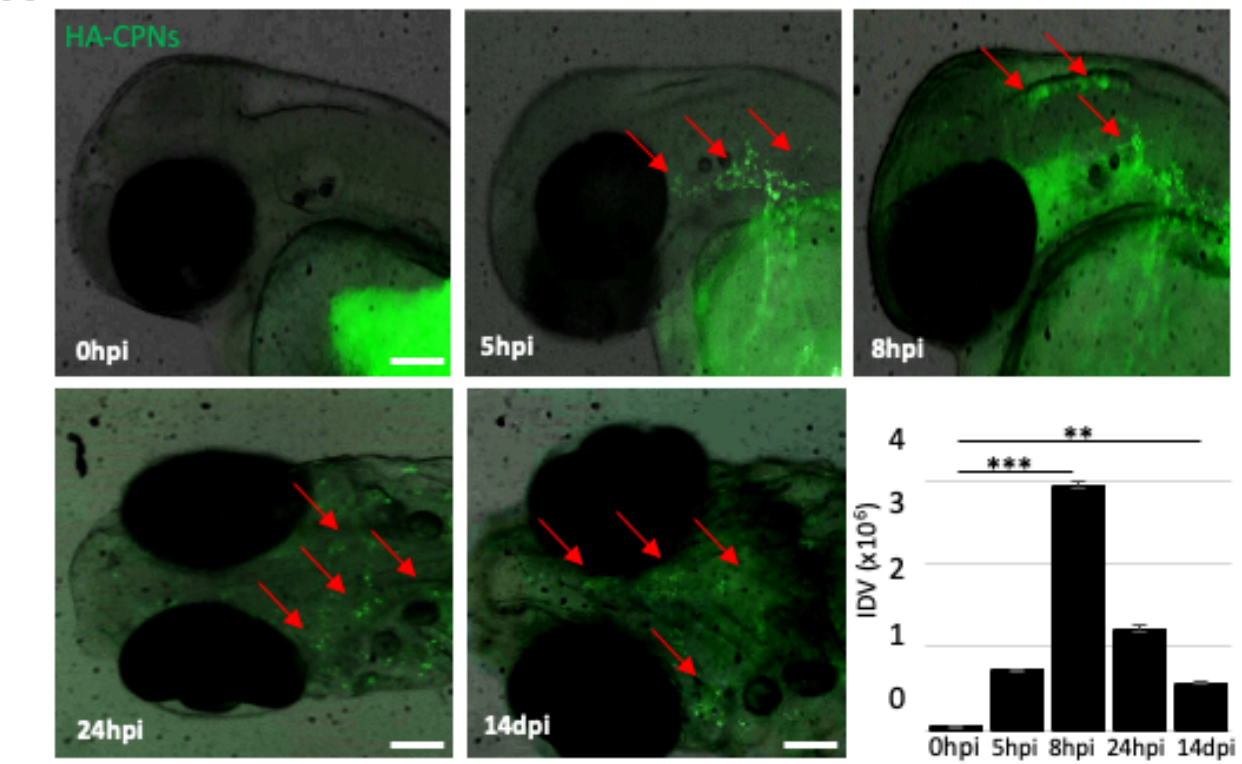

B
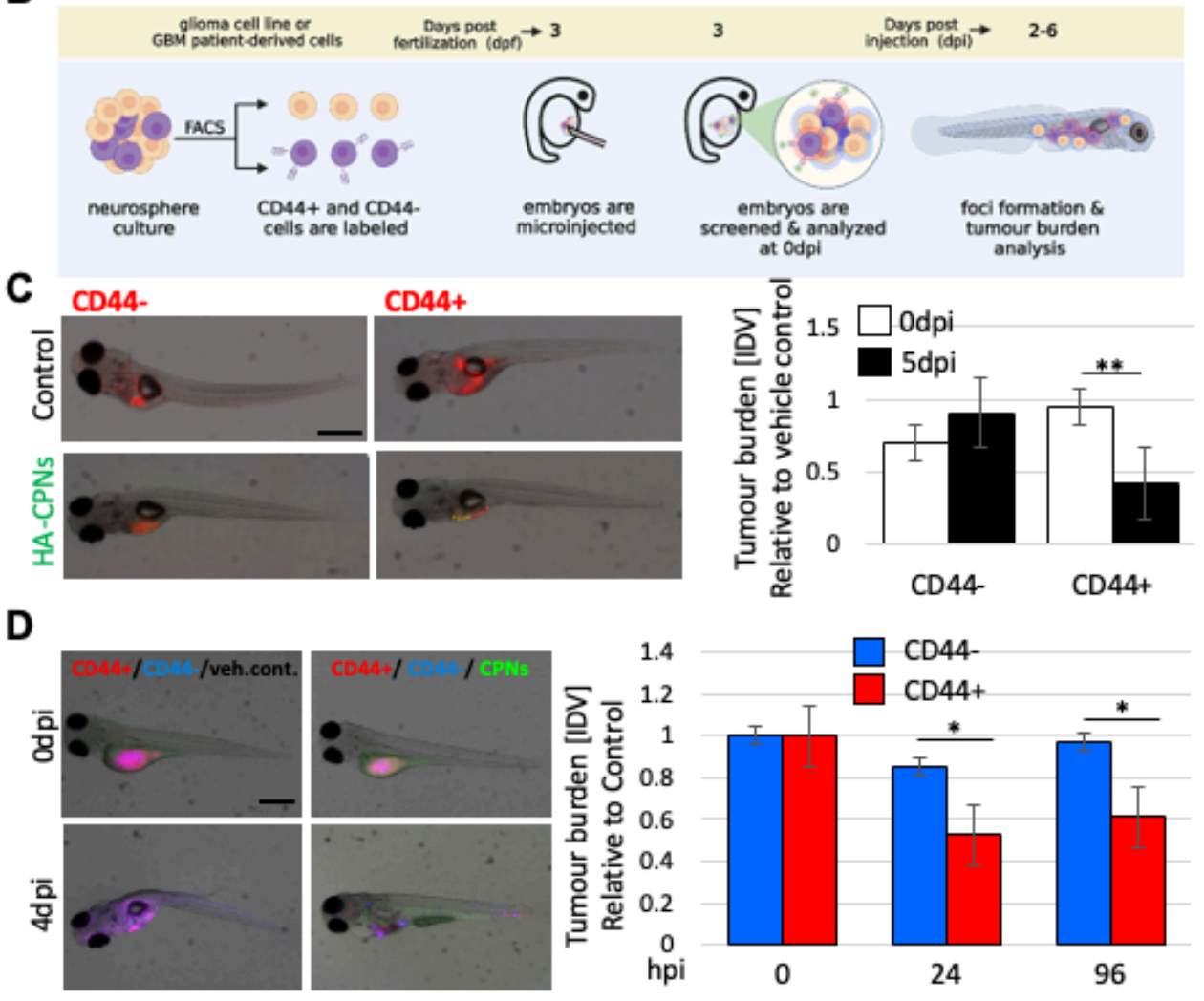

Fig. 5 In vivo effects of HA-CPNs. a HA-CPN blood-brain barrier penetration in zebrafish over a time course of 14 days. Zebrafish injected with 9.2nl of HA-CPNs at 0dpi and imaged at the indicated time points. HA-CPNs detected in the head/brain regions (left) and specific fluorescence 
quantified as IDV (Integrated Density Values) using ImageJ (right). Scale bar $=100 \mu \mathrm{m}$. b Schema of the workflow and timing of zebrafish GBM PDX models. Biorender. c\&d Representative images (left) and tumour foci burden analysis (right) in zebrafish embryos injected with fluorescently labeled U-251 MG cells in the presence of HA-CPNs and/or HA-CPN vehicle control (Control) at 2dpf. c Embryos injected separately with CD44+ and/or CD44- cells and analysed at day 5 dpi. d Embryos co-injected with CD44+ and CD44- cells and analysed at 24 hours, 4 days and 6 days post injection. Tumour burden quantified and graphed as Integrated Density Values (IDV) for HA- CPN treatment/ HA-CPN vehicle control treatment (Control) at the indicated time points. Scale bars: $500 \mu \mathrm{m}$. Data shown as mean $\pm \mathrm{s} . \mathrm{d}, \mathrm{n}=3,{ }^{*} \mathrm{p}<0.05,{ }^{* *} \mathrm{p}<0.01$; Student's $t$-test.

\section{HA-CPNs decrease tumour burden in zebrafish PDX models of human GBM and enhance}

therapeutic response. To verify the anti-glioma properties of HA-CPNs in vivo, we generated zebrafish PDX models using two individual GBM patient- derived cultures. Both, heterogeneous populations of cells and/or FACS sorted CD44- positive and negative cells were employed. Consistently with U251 MG in vivo data, we found that in comparison to the vehicle control, HACPNs were significantly decreasing tumour burden in zebrafish PDX models established using heterogenous patient lines, over a time course of 6 days post injection (Fig.6a). Although we observed tumour growth upregulation over time, in zebrafish PDX models derived using CD44 enriched cell populations treated with both the vehicle control as well as HA-CPNs, the latter demonstrated significantly decreased tumour burden at 4dpi and 6dpi (Fig.6b) suggesting that HACPNs could potentially be combined with other therapeutics to improve the treatment response. Unpublished data from our lab revealed that exposure of U251 MG cells to $15 \mathrm{nM}$ CDK inhibitor, dinaciclib, enriches the culture for CD44+ cells (not shown). To assess if dinaciclib could aid in 
selectivity of HA-CPNs in glioma in vitro, the nanoparticle uptake was assessed in U251 MG cells treated with dinaciclib in comparison to the vehicle control. At the 72-hour timepoint there was a significant increase in the number of HA-CPN positive cells in both the drug treatment and the control, as compared to the earlier 24- and 48- hour time points; however, the dinaciclib treated cells demonstrated significantly higher uptake of HA-CPNs than vehicle treated cultures at 48 and 72 hours (Fig.6c). In vitro drug screening assay conducted using three patient derived cultures (HF3035, HF2927, HF2303) demonstrated that treatment with standard of care Temozolomide (TMZ) alone did not affect the combined viability of the lines tested when compared to the vehicle control (TMZ vehicle). A significant decrease in viability was observed in cells treated with standard of care Temozolomide (TMZ) in triple combination with dinaciclib (DINA) and HACPNs, in comparison to dual treatment with TMZ+ dinaciclib, TMZ+ HA-CPNs and/or dinaciclib+ HA-CPNs, in three lines combined (Fig.6d). This data suggests a potential therapeutic benefit from applying HA-CPNs in combination with known therapeutics against glioma especially in tumours resistant to the available standard of care treatment. 

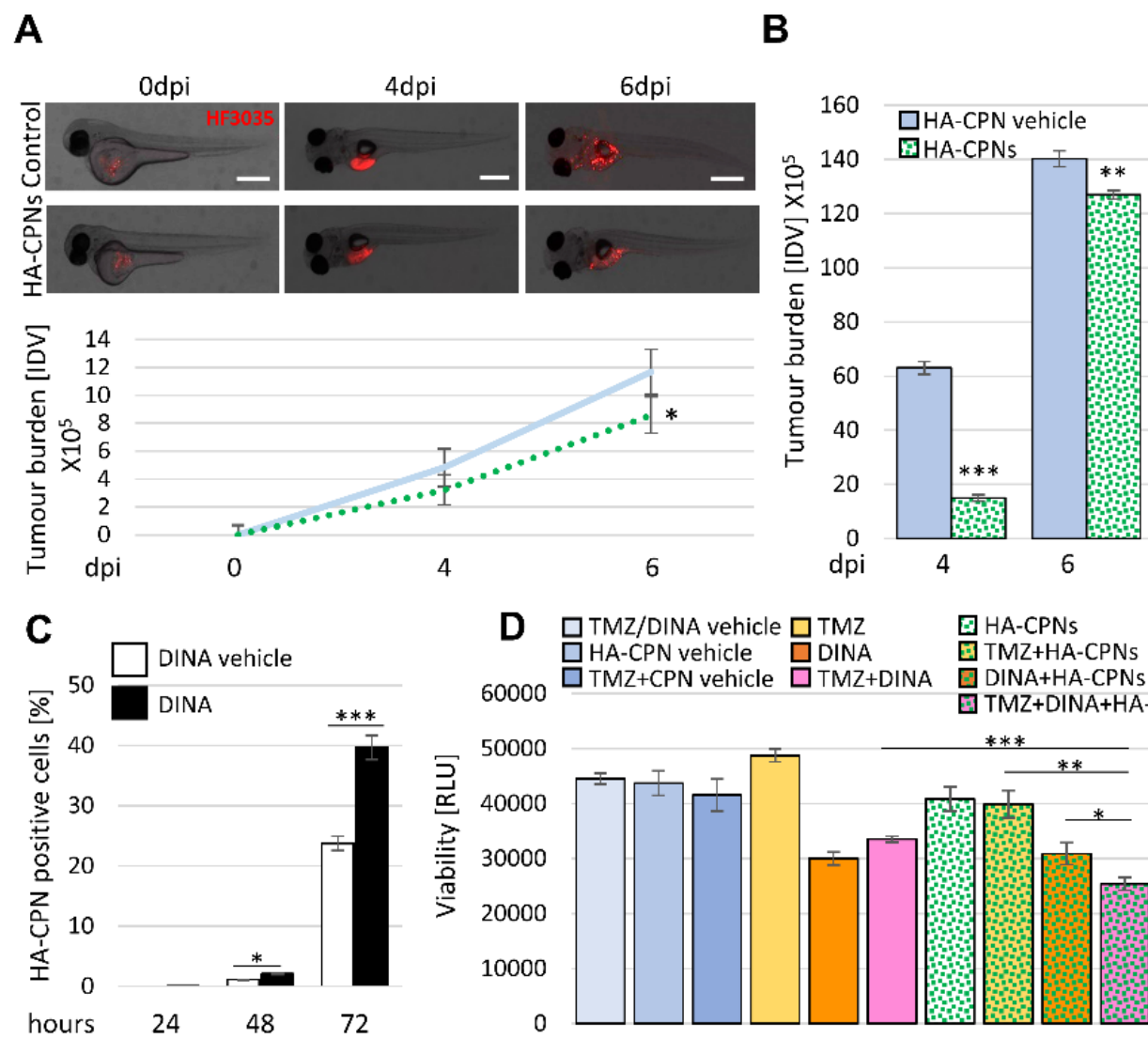

GTMZ+HA-CPNs

GTMZ+DINA+HA-CPNS

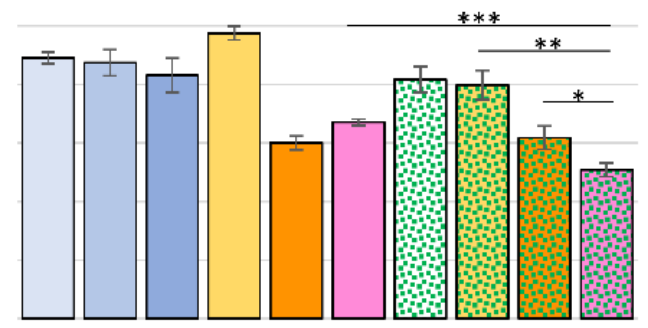

Fig 6 Decrease of tumour burden in GBM zebrafish PDXs and enhanced treatment sensitivity upon exposure to HA-CPNs. a HF3035 and HF2303 GBM patient-derived zebrafish PDX models treated with HA-CPNs compared to HA-CPN vehicle control; representative images of PDX using HF3035 (top) and average tumour burden quantified using ImageJ as IDV at the indicated timepoints (bottom) over two GBM patient lines. b Average tumour burden quantification at the indicated timepoints in PDXs derived from CD44+ HF3035 and CD44+ HF2303 GBM lines treated with HA-CPNs compared to HA-CPN vehicle control (Control) over time. c Cells treated with dinaciclib and/or vehicle control (Control) in the presence of 24 HACPNs/cell. Nanoparticle uptake analysed using flowcytometry at the indicated time points. d Cell viability quantified using a luminescent at 72 hours post HA-CPN and drug treatment, as 
monotherapy or in combination as indicated (TMZ, Temozolomide; DINA, dinaciclib). Data shown as mean $\pm \mathrm{s} . \mathrm{d}, \mathrm{n}=3,{ }^{*} \mathrm{p}<0.05,{ }^{*} \mathrm{p}<0.01,{ }^{* *} \mathrm{p}<0.001$; Student's $t$-test.

\section{Discussion}

Desperate search for novel effective therapeutics against the deadliest forms of cancer has integrated several distinct disciplines in recent years. Materials chemistry is a fast-growing field of chemical research which, by offering avant-garde treatment concepts, changes the mindset of how the disruptive action against cancer cells can be executed. Eradication of extremely heterogeneous and dynamic types of cancer such as GBM calls for multimodal targeting strategies. Novel conjugated polymer nanoparticles bearing hyaluronic acid as peripheral recognition moieties were successfully prepared for selective targeting of GBM. Although, advantages of HA addition to nano-based systems have been reported previously with inorganic and organic particles ${ }^{32}$ this novel system is the first to selectively target aggressive cell populations in human glioma and to demonstrate an effect of CPN accumulation on cell proliferation and in vivo tumour burden.

The HA-CPNs were structurally characterized using advanced characterization techniques for nanoparticles, including TEM, DLS, and SANS. We successfully confirmed that the HA-CPNs are spherical and have a relatively uniform size distribution, as confirmed by low dispersity index. Their average diameter of $109 \mathrm{~nm}$ maximizes their potency as theranostic agents to be transitioned across the BBB. ${ }^{33}$

This study optimized biological application of HA-CPNs using human glioma U-251 MG cell line and validated the behavior of HA-CPNs in an in vitro and in vivo biologically relevant setting using GBM cultures obtained at surgery from individual GBM patients. Assessment of the 
incubation time, along with the concentration of nanoparticles are essential to properly devise the parameters to the biological system and allowed us to standardize the concentration used across several experiments. We found HA-CPN uptake to be dependent on both time and CPN concentration. Nanoparticle uptake heavily depends on several changes occurring at the cell membrane including but not limited to electrostatic interactions between nanoparticle and the membrane, ${ }^{34}$ hydrophobic and interfacial forces, ${ }^{35,36}$ as well as the type and efficiency of the endocytotic mechanism responsible for the internalization of the nanoparticles ${ }^{37}$ Hence, uptake of nanoparticles can fluctuate in diverse growth settings. ${ }^{38,39}$ We demonstrate that HA-CPN uptake varies at different phases of the cell cycle, and that cells permitted to undergo cell cycle progression through G1-S-G2 were able to accumulate the highest number of HA-CPNs. This may support that HA-CPNs will accumulate in more rapidly dividing tumourigenic cell populations. Our results are with other studies that have shown that different cell cycle phases internalize nanoparticles at different rates with the highest uptake occurring at $\mathrm{G} 2 / \mathrm{M}$, followed by $\mathrm{S}$, and then $\mathrm{G} 0 / \mathrm{G} 1$ phase. ${ }^{31,40}$

Importantly, this study explored the specificity of HA-CPNs in targeting glioma initiating cells which are positive for CD44 receptor. To determine whether the CD44 ligand, HA, present on CPNs plays a role in the nanoparticle selectivity, the U-251 MG cells and/or patient primary lines were sorted via FACS to obtain CD44+ and CD44- cell populations. Alternatively, heterogeneous population of U-251 MG cells was treated with HA-CPNs and then stained with a fluorescent antibody and co-expression of both signals was measured by flow cytometry. Both approaches demonstrated a significant increase of uptake in CD44+ cells as compared to CD44cell populations by U-251 MG cells as well in four out of five primary GBM cultures tested. 
Several studies have shown that HA-receptor interaction promotes glioma proliferation and invasiveness, and abrogation of the interaction leads to diminished glioma aggressiveness. ${ }^{41-}$ ${ }^{43}$ Hence it was important to determine if HA-CPNs would trigger any biological activation of the CD-44 receptor. Surprisingly, we show that treatment with increasing concentrations of HA-CPNs for prolonged time leads to a downregulation of proliferation, metabolic activity and stemness in GBM-patient derived cultures. We show that exposure to HA-CPNs decreases migration in collective as well as single cell invasion of GBM primary cultures. Our results support that the HA-CPNs do not activate CD44 proliferative signaling and may alternatively either inhibit CD44 or have cytotoxic properties on the cells that they accumulate. Dissecting the molecular mechanism behind the HA-CPN- mediated effects in of high priority. In vivo investigation into the biological effects of HA-CPNs using zebrafish revealed selective growth inhibition of the CD44+ but not CD44-tumour foci over time, either in individual xenografts or using CD44+/CD44- co-injection model. Consequently, using zebrafish PDX model we demonstrate that targeting of the CD44+ populations in vivo results in significantly abrogated growth of tumour bulk supporting the efficacy of therapeutic strategies targeting TICs in glioma. Providing treatment solutions for tumours presenting therapy resistance is of highest demand. Our results in vitro show that combining standard of care TMZ therapy with an agent selective for CD44 cell populations and with HACPN treatment successfully abrogates viability of TMZ- resistant GBM cells validating this nanoparticle system for further testing in pre-clinical models.

CD44 expression has been correlated with GBM aggressiveness and poor patient prognosis ${ }^{11}$ suggesting that targeting of $\mathrm{CD} 44+$ populations may have most significant effect on the clinical outcomes in patients cohorts of reported signature upregulation of CD44 expression levels, such as patients with the mesenchymal GBM subtype. ${ }^{44}$ Although statistically significant 
results obtained collectively in five patient lines seem promising, not all GBM tumours are driven by initiating cells that are CD44 positive: despite CD44 receptor selected for proof of principle in this study, the versatility of the CPN system allows for future modification and elaboration of personalized approaches. A careful, multi-assay evaluation provided in this study demonstrates that diketopyrrolopyrrole-based conjugated polymers, generated with HA, can selectively target CD44+ cells in tested GBM patient- derived cultures and exert anti-tumourigenic properties in zebrafish PDX model in vivo. Our data suggest that further validation of this system and its potential versatility can appoint a novel targeting therapy against glioblastoma.

\section{Methods}

Details on P(DPP-T) synthesis and the instruments used for its characterization can be found in the Supplementary Information.

Nanoprecipitation. $1 \mathrm{mg}$ of $\mathrm{P}(\mathrm{DPP}-\mathrm{T})$ conjugated polymer and $2 \mathrm{mg}$ of the fluorescein hyaluronic acid surfactant were dissolved in $1 \mathrm{~mL}$ of tetrahydofuran (THF) and left to stir for 30 minutes. The solution was then injected in $6 \mathrm{~mL}$ of deionized water under probe sonication (amplitude of 60 , power of $55 \mathrm{~W}$ for 5 minutes). After sonication, the sample was passed through a $0.2 \mu \mathrm{m}$ PTFE (hydrophobic) syringe filter to remove any large aggregated materials. The nanoparticles were washed multiple times with deionized water and the resulting aqueous suspension was stored at $4^{\circ} \mathrm{C}$ prior to use. The vehicle control and Polysorbate-containing conjugated polymer nanoparticles (Nc-CPN) were prepared via analogous protocols.

Cell Culture and HA-CPN treatment. U-251 MG wt cells were obtained from (Dr. Rutka, SickKids Hospital, Toronto). The cells were cultured at $37^{\circ} \mathrm{C}$ and $5 \% \mathrm{CO}_{2}$ in Minimum Essential Medium Eagle (EMEM), (Sigma-Aldrich) supplemented with 10\% Fetal Bovine Serum (FBS) 
(Gibco, \#10437028), 1\% Penicillin and Streptomycin (Invitrogen, \#15140148), 1mM sodium pyruvate, and with 1x non-essential amino acids (NEAA) (Sigma, \#M7145). CPNs were added directly to the media in volume corresponding to the desired number of $\mathrm{CPNs} /$ cell concentration, THF-evaporated water was used as vehicle control in corresponding volumes. For the proliferation assay the concentration of $\mathrm{CPNs} /$ cell was varied and included 5, 10, 24, 37, and $53 \mathrm{CPNs} /$ cell $(10 \mu \mathrm{L}, 20 \mu \mathrm{L}, 45 \mu \mathrm{L}, 70 \mu \mathrm{L}$, and $100 \mu \mathrm{L}$ of CPNs or the vehicle control) per $2 \mathrm{~mL}$ of growth media. For the MTT assay the applied concentrations of CPNs/cell included 6, 12, 24, 48, and 63 CPNs/cell $(3 \mu \mathrm{L}, 7.5 \mu \mathrm{L}, 15 \mu \mathrm{L}, 30 \mu \mathrm{L}$, and $45 \mu \mathrm{L}$ of CPN solution or vehicle control). The cultures treated with CPNs were protected from light. For immunocytochemistry, cells were cultured on coverslips for 2, 4, 7, and 24-hours with $24 \mathrm{CPNs} / \mathrm{cell}$.

GBM patient- derived cultures. Resected tumors were collected at the Henry Ford Hospital, with written consent from patients in accordance with institutional guidelines as approved by the Institutional Review Board at Henry Ford Hospital. Pathology was graded according to the WHO criteria. The cells were extracted and cultured as spheres as described previously. ${ }^{45}$

Cell Cycle Synchronization. Double thymidine block was performed using $2 \mathrm{mM}$ thymidine for 18 hours. At the 18-hour mark, the media was replaced with regular growth media and incubated for 9-hours before thymidine was re-added to the plates for another 18-hour incubation. Cell starvation included 24-hour incubation of cells in media supplemented with 1\% FBS. Mitotic population enrichment was achieved by incubation of cultures with $2 \mathrm{pg} / \mathrm{mL}$ nocodazole for 24 hours.

Flow Cytometry. Single cell suspension $\left(10^{6}\right.$ cells $\left./ \mathrm{mL}\right)$ in PBS with $2 \mathrm{mM}$ EDTA were analyzed using BD LSR Fortessa ${ }^{\mathrm{TM}}$ X-20 (BD) flow cytometer (Becton Dickinson). FITC laser was used to 
detect CPN positive cells and APC laser detected cells labeled with APC conjugated CD44 antibody. Propidium iodide was used as nuclear control.

Cell Sorting. One million cells were stained with anti CD44 APC-conjugated antibody (BD, $\# 559942,20 \mu 1$ per $10^{6}$ cells in $100 \mu 1$ of stain buffer) on ice for 45 minutes, washed and suspended in $\mathrm{PBS} / 2 \mathrm{mM}$ EDTA at the concentration of $10^{6}$ cells $/ \mathrm{mL}$. The cells were sorted using BD FACSAria Fusion ${ }^{\mathrm{TM}}$ cell sorter (BD) to separate CD44+ from CD44- cells and/or Ha-CPN+ and HA-CPN- cells.

Proliferation Assay. Cells were seeded at density of $5 \times 10^{3}$ cells per well in 6 well plates. Number of viable cells was assessed at the indicated time points (2-, 4-, and 6-days post-seeding) using trypan blue exclusion assay via hemocytometer counts. Total numbers of viable cells were graphed.

Neurosphere formation assay. Single cell suspension of GBM patient derived cells was seeded at $5 \times 10^{4}$ in 12 well plates containing $1.5 \mathrm{~mL}$ growth media. HA-CPNs were applied at a concentration of $24 \mathrm{CPNs} / \mathrm{cell}(150 \mu \mathrm{L})$ and plates were incubated at $37{ }^{\circ} \mathrm{C}$ and $5 \% \mathrm{CO}_{2}$. After 6 days, spheres were imaged at 10x magnification under five different fields of view using a Leica inverted microscope (Leica CTR 6500). Primary neurospheres were dissociated in PBS pH 7.4 (ThermoFisher, \#10010-023) and seeded into a secondary generation following the same procedure. ImageJ analysis was used for quantification of sphere diameter and number.

qRT-PCR. Total RNA was extracted from cells utilizing RNeasyPlus Mini Kit (Qiagen) and reverse transcribed using qScript cDNA SuperMix (Quanta), according to manufacturer instructions. For each experiment, samples were reverse transcribed at the same time and cDNA was stored at $-20^{\circ} \mathrm{C}$. Real time qPCR with SYBR ${ }^{\mathrm{TM}}$ Green (Applied Biosystems) fluorescent 
detection was performed using Viia7 thermocycler (Life Technologies). Data was analyzed using Viia7 software and represented as RQ relative to control. Primer sequences were:

Sphere invasion assay. Single cell suspension of $2.5 \times 10^{4}$ human primary cells were seeded in 96 well round bottom ultra-low attachment plates containing $200 \mu \mathrm{L}$ growth media per well. Spheroids were cultured at $37^{\circ} \mathrm{C}$ and $5 \% \mathrm{CO}_{2}$ for 3 days. Individual spheroids were embedded in a $30 \mu \mathrm{L}$ droplet of Matrigel $^{\mathrm{TM}}$ (FisherScientific, \#CB40234C) mixed prior with HA-CPN volume corresponding to 12-24 HA-CPNs/cell. Embedded spheroids were transferred to 24 well surface repellent plates containing $750 \mu \mathrm{L}$ growth media and imaged every 24 hours using a Leica inverted microscope (Leica CTR 6500). The average radius of collective cell migration (4 measurements per sphere, 6 spheres per treatment over 3 patient lines) and single cell migration were measured using ImageJ, starting from the central point of the core in each sphere analyzed. The radius of collective cell migration was corrected by the radius of time 0 . ImageJ was used to quantify number of migrating single cells.

Migration assay. Cells were seeded at $7.5 \times 10^{4}$ into twelve well cell culture inserts with $8 \mu \mathrm{m}$ porosity polyester membrane filters (VWR, \#62406-176) in 500 $\mu \mathrm{L}$ of serum-free EMEM. $1 \mathrm{~mL}$ of growth media was added into each well of the plate. After a 24 -hour incubation period at $37^{\circ} \mathrm{C}$ and $5 \% \mathrm{CO}_{2}$, not migrated cells were removed from the insert using a cotton swab. Migrated cells which crossed the membrane were washed in $1 \times$ PBS and fixed in 4\% PFA, followed by staining with $0.1 \%$ crystal violet in methanol. Rinsed membranes were imaged at $10 \mathrm{x}$ magnification under five different fields of view using a Leica inverted microscope (Leica CTR 6500). ImageJ analysis provided quantification of the signal's Integrated Density.

Drug treatment assay. Human primary cells were cultured at $1 \times 10^{4}$ cells per well in 96 well round bottom ultra-low attachment plates containing $100 \mu \mathrm{L}$ growth media. Temozolomide $(20 \mu \mathrm{M})$ 
(Selleckchem, \#S1237), dinaciclib (15nM) (Selleckchem, \#S2768) and HA-CPNs (24 CPNs/cell) were applied 1-hour post-seeding. Respective DMSO and HA-CPN vehicle controls of each drug were used. Plates were incubated at $37{ }^{\circ} \mathrm{C}$ and $5 \% \mathrm{CO}_{2}$ for 72 -hours and all treatments were refreshed at half of the originally applied concentration every 24 hours throughout incubation period. Cell viability was measured via the CellTiter-Glo 3D Cell Viability Assay (Promega) using Spectramax plate reader.

MTT Assay. Cells were seeded in $96-$ well plates $\left(5 \times 10^{3}\right.$ per well in $100 \mu \mathrm{L}$ of growth media). The plates were protected from light with aluminum foil and incubated at $37^{\circ} \mathrm{C}$ and $5 \% \mathrm{CO}_{2}$. At the indicated time points, the media was removed and replaced with $20 \mu \mathrm{L}$ of MTT solution $(5 \mathrm{mg} / \mathrm{mL}$ thiazolyl blue tetrazolium bromide along with filter-sterilized PBS) (Sigma Aldrich, \#M5655) and the plates were incubated for $2-4$ hours at $37^{\circ} \mathrm{C}$ and $5 \% \mathrm{CO}_{2}$. This was followed by the addition of $100 \mu \mathrm{L}$ of extraction buffer. The plates were incubated overnight at $37^{\circ} \mathrm{C}$ and $5 \% \mathrm{CO}_{2}$ and protected from light. The absorbance at 590nm was measured using SpectraMax plate reader.

Dinaciclib Mediated CD44 Enrichment and CPN Uptake Analysis. Cells $\left(2.5 \times 10^{4}\right)$ were seeded in $6 \mathrm{~cm}$ plates and treated with 15nM dinaciclib (Selleckchem, \#S2768) or DMSO (as a vehicle control), and $150 \mu \mathrm{L}$ of $\mathrm{CPNs}$ for 72 -hours at $37^{\circ} \mathrm{C}$ and $5 \% \mathrm{CO}_{2}$ while protected from light. The media was replaced on each of these plates, along with the addition of drugs and CPNs every 24 hours. At 72 hours the cells were subjected to flowcytometry analysis.

Immunocytochemistry Analysis. The cells were washed twice with 1xPBS, followed by fixing in 4\% PFA and staining with anti- CD44 antibody (Novus, \#NBP1-31488) when required, for 2 hours. The cells were then washed with 1xPBS and HBSS and incubated with fluorophore conjugated secondary antibody (ThermoFisher, \#A-11011) for 1 hour and Hoechst nuclear stain for 15 minutes. The coverslips were washed for 5 minutes each, with HBSS, 1xPBS, and diH2O. 
The coverslips were mounted on microscope slides using aqueous mounting media and imaged using a Leica inverted microscope (Leica CTR 6500 microscope).

Zebrafish Injections and PDX models. Zebrafish embryos at $3 \mathrm{dpf}$ were anesthetized and positioned on the $1 \%$ agarose. Cells $\left(10^{6}\right.$ cells $\left./ \mathrm{mL}\right)$ were labeled with Vibrant ${ }^{\mathrm{TM}}$ DiI and DiD cell tracing dyes (ThermoFisher, \# V22885, V22887) and 9.2nl of cell suspension in the presence of CPNs (4 CPNs/cell) or vehicle control were injected into the midsection of the yolk sac using Nanoject II Auto- Nanoliter Injector (Drummond). The embryos were imaged at the indicated timepoints using Leica fluorescence stereomicroscope M205. Analysis of tumour foci burden was measured, and Integrated Density of the signal was quantified using ImageJ software. The values obtained from CPN treatment were corrected by the vehicle control values at each time point.

\section{References}

1. Adamson, C. et al. Glioblastoma multiforme: a review of where we have been and where we are going. Expert Opin. Investig. Drugs 18, 1061-1083 (2009).

2. Wipfler, K., Cornish, A. S. \& Guda, C. Comparative molecular characterization of typical and exceptional responders in glioblastoma. Oncotarget 9, 28421-28433 (2018).

3. Miroshnikova, Y. A. et al. Tissue mechanics promote IDH1-dependent HIF1 $\alpha$-tenascin C feedback to regulate glioblastoma aggression. Nat. Cell Biol. 18, 1336-1345 (2016).

4. Bastola, S. et al. Glioma-initiating cells at tumor edge gain signals from tumor core cells to promote their malignancy. Nat. Commun. 11, 1-17 (2020).

5. Pesenti, C. et al. The genetic landscape of human glioblastoma and matched primary 
cancer stem cells reveals intratumour similarity and intertumour heterogeneity. Stem Cells Int. 2019, (2019).

6. Gimple, R. C., Bhargava, S., Dixit, D. \& Rich, J. N. Glioblastoma stem cells: Lessons from the tumor hierarchy in a lethal cancer. Genes Dev. 33, 591-609 (2019).

7. Dirkse, A. et al. Stem cell-associated heterogeneity in Glioblastoma results from intrinsic tumor plasticity shaped by the microenvironment. Nat. Commun. 10, 1-16 (2019).

8. Cohen, Z. R. et al. Localized RNAi therapeutics of chemoresistant grade IV glioma using hyaluronan-grafted lipid-based nanoparticles. ACS Nano 9, 1581-1591 (2015).

9. Klank, R. L. et al. Biphasic Dependence of Glioma Survival and Cell Migration on CD44 Expression Level. Cell Rep. 18, 23-31 (2017).

10. Wolf, K. J. et al. A mode of cell adhesion and migration facilitated by CD44-dependent microtentacles. Proc. Natl. Acad. Sci. U. S. A. 117, (2020).

11. Si, D., Yin, F., Peng, J. \& Zhang, G. High expression of CD44 predicts a poor prognosis in glioblastomas. Cancer Manag. Res. 12, 769-775 (2020).

12. Hou, C. et al. Expression of CD44 and the survival in glioma: A meta-analysis. Biosci. Rep. 40, 1-10 (2020).

13. Harder, B. G. et al. Developments in Blood-Brain Barrier Penetrance and Drug Repurposing for Improved Treatment of Glioblastoma. Front. Oncol. 8, 1-10 (2018).

14. Arvanitis, C. D., Ferraro, G. B. \& Jain, R. K. The blood-brain barrier and blood-tumour barrier in brain tumours and metastases. Nat. Rev. Cancer 20, 26-41 (2020). 
15. Miao, R., Xia, L. Y., Chen, H. H., Huang, H. H. \& Liang, Y. Improved Classification of Blood-Brain-Barrier Drugs Using Deep Learning. Sci. Rep. 9, 1-11 (2019).

16. Wang, D., Wang, C., Wang, L. \& Chen, Y. A comprehensive review in improving delivery of small-molecule chemotherapeutic agents overcoming the blood-brain/brain tumor barriers for glioblastoma treatment. Drug Deliv. 26, 551-565 (2019).

17. Kim, S. et al. Conjugated polymer nanoparticles for biomedical in vivo imaging. Chem. Commun. 46, 1617-1619(2010).

18. Wang, Y., Feng, L. \& Wang, S. Conjugated Polymer Nanoparticles for Imaging, Cell Activity Regulation, and Therapy. Adv. Funct. Mater. 29, 1-20 (2019).

19. $\mathrm{Pu}, \mathrm{K}$. et al. Semiconducting polymer nanoparticles as photoacoustic molecular imaging probes in living mice. Nat. Nanotechnol. 9, 233-239 (2014).

20. Repenko, T. et al. Bio-degradable highly fluorescent conjugated polymer nanoparticles for bio-medical imaging applications. Nat. Commun. 8, 8-15 (2017).

21. Guo, B. et al. Molecular Engineering of Conjugated Polymers for Biocompatible Organic Nanoparticles with Highly Efficient Photoacoustic and Photothermal Performance in Cancer Theranostics. ACS Nano 11, 10124-10134 (2017).

22. Wei, Z. et al. Donor-acceptor conjugated polymer-based nanoparticles for highly effective photoacoustic imaging and photothermal therapy in the NIR-II window. Chem. Commun. 56, 1093-1096 (2020).

23. Zhang, B. et al. EGFP-EGF1-conjugated nanoparticles for targeting both neovascular and 
glioma cells in therapy of brain glioma. Biomaterials 35, 4133-4145 (2014).

24. Zhong, Y., Meng, F., Deng, C. \& Zhong, Z. Ligand-directed active tumor-targeting polymeric nanoparticles for cancer chemotherapy. Biomacromolecules 15, 1955-1969 (2014).

25. Feng, X. et al. Multi-targeting Peptide-Functionalized Nanoparticles Recognized Vasculogenic Mimicry, Tumor Neovasculature, and Glioma Cells for Enhanced Antiglioma Therapy. ACS Appl. Mater. Interfaces 7, 27885-27899 (2015).

26. Choi, K. Y. et al. Hyaluronic Acid-Based Activatable Nanomaterials for StimuliResponsive Imaging and Therapeutics: Beyond CD44-Mediated Drug Delivery. Adv. Mater. 31, 1-18 (2019).

27. Xu, Y. et al. Lactoferrin-coated polysaccharide nanoparticles based on chitosan hydrochloride/hyaluronic acid/PEG for treating brain glioma. Carbohydr. Polym. 157, 419-428 (2017).

28. Zhang, S. et al. The Critical Role of Electron-Donating Thiophene Groups on the Mechanical and Thermal Properties of Donor-Acceptor Semiconducting Polymers. $A d v$. Electron. Mater. 1800899 (2019).

29. Hammouda, B. A new Guinier-Porod model. J. Appl. Crystallogr. 43, 716-719 (2010).

30. Shah, R. M. et al. Structure Analysis of Solid Lipid Nanoparticles for Drug Delivery: A Combined USANS/SANS Study. Part. Part. Syst. Charact. 36, 1-7 (2019).

31. Kim, J. A., Aberg, C., Salvati, A. \& Dawson, K. A. Role of cell cycle on the cellular 
uptake and dilution of nanoparticles in a cell population. Nat. Nanotechnol. 7, 62-68 (2012).

32. Játiva, P. \& Ceña, V. Use of nanoparticles for glioblastoma treatment: A new approach. Nanomedicine 12, 2533-2554 (2017).

33. Juillerat-Jeanneret, L. The targeted delivery of cancer drugs across the blood-brain barrier: chemical modifications of drugs or drug-nanoparticles? Drug Discov. Today 13, 1099$1106(2008)$.

34. Forest, V. \& Pourchez, J. Preferential binding of positive nanoparticles on cell membranes is due to electrostatic interactions: A too simplistic explanation that does not take into account the nanoparticle protein corona. Mater. Sci. Eng. C 70, 889-896 (2017).

35. Wiegand, T. et al. Forces during cellular uptake of viruses and nanoparticles at the ventral side. Nat. Commun. 11, 1-13 (2020).

36. Desmet, C. et al. Characterisation of nanomaterial hydrophobicity using engineered surfaces. J. Nanoparticle Res. 19, 117 (2017).

37. Behzadi, S. et al. Cellular uptake of nanoparticles: journey inside the cell. Chem. Soc. Rev. 46, 4218-4244 (2017).

38. Moore, T. L. et al. Nanoparticle administration method in cell culture alters particle-cell interaction. Sci. Rep. 9, 1-9 (2019).

39. Ma, L., Kohli, M. \& Smith, A. Nanoparticles for combination drug therapy. ACS Nano 7, 9518-9525 (2013). 
40. Rees, P., Wills, J. W., Brown, M. R., Barnes, C. M. \& Summers, H. D. The origin of heterogeneous nanoparticle uptake by cells. Nat. Commun. 10, 1-8 (2019).

41. Gilg, A. G. et al. Targeting hyaluronan interactions in malignant gliomas and their drugresistant multipotent progenitors. Clin. Cancer Res. 14, 1804-1813 (2008).

42. Pogoda, K. et al. Soft Substrates Containing Hyaluronan Mimic the Effects of Increased Stiffness on Morphology, Motility, and Proliferation of Glioma Cells. Biomacromolecules 18, 3040-3051 (2017).

43. Koh, I. et al. The mode and dynamics of glioblastoma cell invasion into a decellularized tissue-derived extracellular matrix-based three-dimensional tumor model. Sci. Rep. 8, 112 (2018).

44. Behnan, J., Finocchiaro, G. \& Hanna, G. The landscape of the mesenchymal signature in brain tumours. Brain 142, 847-866 (2019).

45. Hasselbach, L. et al. Optimization of High Grade Glioma Cell Culture from Surgical Specimens for Use in Clinically Relevant Animal Models and 3D Immunochemistry. $J$. Vis. Exp. 83, 51088 (2014).

\section{Acknowledgements}


This study was financially supported by a Seeds4Hope grant from the Windsor Cancer Centre Foundation and administered by WE-SPARK Health Institute and the University of Windsor. This work was also supported by NSERC through a Discovery Grant (S.R.-G., RGPIN-2017-06611) and CIHR Project Grant \#406811 (L. A. P.). S.R.-G. also acknowledges the Canada Foundation for Innovation (CFI) and Ontario Research Fund, and the University of Windsor for financial support. Access to NGB-30m SANS was provided by the Centre for High Resolution Neutron Scattering, a partnership between the National Institute of Standards and Technology and the National Science Foundation under Agreement No. DMR-2010792. We acknowledge the support of the National Institute of Standards and Technology, U.S. Department of Commerce, in providing the neutron research facilities used in this work and the technical support of Elizabeth G. Kelley. The authors would also like to acknowledge the Windsor-Essex residents who have made this research possible through their generous donations.

\section{Authors Contributions}

S.R-G., L.A.P., D.L. and G.T.M. conceived and directed the project. G.T.M., A.A., A.M. and S. R.-G. synthesized and characterized the HA-CPNs and their precursors by DLS and TEM. M.D. and D.M. performed SANS measurements and data analysis. D.L., S.A. and F.N. performed the complete biological evaluation of the CPNs both in vitro and in vivo, collected all data and performed the analysis. M.S., A.D., A.S., S.K., provided support in obtaining primary GBM patirnt cultures. D.L., G.T.M., L.A.P. and S.R.-G. wrote the manuscript, and all the coauthors commented on the manuscript.

\section{Author Information}


The authors declare no competing financial interests. Readers are welcome to comment on the online version of the paper. Correspondence and requests for materials should be addressed to L.A.P (lporter@uwindsor.ca) or S.R.-G. (srondeau@uwindsor.ca) 